\title{
Implantação da vacina Bacilo Calmette-Guérin (BCG) nas maternidades do município de Porto Alegre - RS, 2019 - 2020
}

\author{
Bacille Calmette-Guérin (BCG) vaccine implementation in maternity hospitals in the city of \\ Porto Alegre - RS, 2019 - 2020 \\ Implementación de la vacuna Bacilo Calmette-Guérin (BCG) en las maternidades de la \\ ciudad de Porto Alegre - RS, 2019 - 2020
}

Renata Lobatto Capponi ${ }^{1 *}$, Ceura Beatriz de Souza Cunha1 ${ }^{1}$ Luiza Pereira MachadoBronca ${ }^{1}$, Letícia Bueno Silva1.

\section{RESUMO}

Objetivo: Descrever os impactos gerados pela implantação da administração da vacina BCG nas maternidades que atendem no município de Porto Alegre - RS, em 2019 e 2020. Métodos: Para sua análise foram utilizados somente dados secundários disponibilizados no Sistema de Informação do Programa Nacional de Imunizações e no Sistema de Insumos Estratégicos. Essas informações foram separadas e tabuladas em uma planilha, utilizando-se o software Microsoft Excel ${ }^{\circledR}$ 2010. Resultados: Os resultados demonstraram que a mudança no fluxo dos serviços vacinadores oportunizou aumento na cobertura vacinal do município, atingindo uma porcentagem de $100 \%$ após a alteração. Com o novo fluxo, a maioria dos recémnascidos encontra-se imunizado até o segundo dia de vida, mais próximo da meta preconizada pelo Ministério da Saúde. Tal resultado mostra-se diferente da realidade encontrada com a centralização dos serviços de administração nas unidades de saúde de referência. Considerações finais: As mudanças realizadas trouxeram um maior acesso à vacina da BCG, aumentando a cobertura vacinal e contribuindo para uma imunização precoce.

Palavras-chave: Bacilo de calmette-guérin, Imunizações, Tuberculose.

\begin{abstract}
Objective: Describe the impacts generated by implantation of the BCG vaccine administration in the city of Porto Alegre - RS, in 2019 e 2020. Methods: For this analysis, were used only secondary data, granted by the Information System of the National Immunization Program and the Strategic Inputs System. Those informations were separated and tabulated in a chart, using Microsoft Excel ${ }^{\circledR}$ 2010. Results: The results demonstrate that the change in the flow of vaccinating services provided an increase in the city's vaccine coverage, reaching a percentage of $100 \%$ after the change. With the new flow, most newborns are immunized until the second day of life, which is closer to the goal established by the Health Ministry of Brazil. This result is different from the reality found with the administration services centralization in reference health units. Conclusion: The changes made brought greater access to the BCG vaccines, increasing the vaccine coverage and contributing to early immunization.
\end{abstract}

Key words: Bacille camette-guérin, Immunizations, Tuberculosis.

\section{RESUMEN}

Objetivo: Este estudio tuvo como objetivo describir los impactos generados por la implementación de la administración de la vacuna BCG en las maternidades que atienden la ciudad de Porto Alegre (RS), 2019 2020. Métodos: Para su análisis, solo se dispone de datos secundarios en el Programa. Se utilizó el Sistema de Información Sistema Nacional de Inmunizaciones e Insumos Estratégicos. Esta información se separó y tabuló en una hoja de cálculo, utilizando el software Microsoft Excel ${ }^{\circledR}$ 2010. Resultados: Los resultados encontrados mostraron que el cambio en el flujo de los servicios de vacunación proporcionó un aumento en la cobertura de vacunación del municipio, alcanzando un porcentaje del $100 \%$ después del cambio. Con el nuevo flujo, la mayoría de los recién nacidos se vacunan hasta el o día de vida, más cerca de la meta

${ }^{1}$ Diretoria de Vigilância em Saúde (DVS), Porto Alegre - RS. *E-mail: renata.capponi@portoalegre.rs.gov.br 
recomendada por el Ministerio de Salud, a diferencia de lo que ocurría con la centralización de los servicios de administración en las unidades de salud de referencia. Conclusión: Los cambios realizados trajeron un mayor acceso a los inmunobiológicos BCG, aumentando la cobertura de la vacuna y contribuyendo a una inmunización lo antes posible.

Palabras clave: Bacillus calmette-guérin, Inmunizaciones, Tuberculosis.

\section{INTRODUÇÃO}

O Programa Nacional de Imunizações (PNI), coordenado pelo Ministério da Saúde, vem se consolidando como um dos mais importantes instrumentos de intervenções nas políticas públicas de saúde. Apesar de ser considerado um programa novo, criado em 1973, conta com uma história de vitórias e muitos desafios. Dentre as várias conquistas realizadas pelo PNI, destaca-se a erradicação da varíola e da transmissão da poliomielite no Brasil, além da diminuição de milhares de casos de hospitalizações e óbitos por outras doenças imunopreveníveis. (DOMINGUES CMAS, et al., 2020).

Uma dessas doenças é a Tuberculose (TB), que apesar de possuir altos índices de casos no país, apresenta uma redução nos casos considerados mais graves. Este fato é atribuído à imunização em massa que ocorre há anos. No Brasil, estima-se que ocorram 73.864 mil casos por ano dessa doença, sendo que o coeficiente de mortalidade foi de 2,15 por 100.000 habitantes no ano de 2018 (BRASIL, 2020). Considerando isso, as estratégias nacionais para o controle da tuberculose têm como meta diagnosticar $90 \%$ dos casos esperados e alcançar a cura em $85 \%$ dos casos diagnosticados (BRASIL, 2002).

Nesse cenário, o Ministério da Saúde tem desenvolvido diversas ações que visam reduzir a morbimortalidade por TB. Dentre elas, com o objetivo de intensificar as ações de prevenção e reduzir o adoecimento, destaca-se a manutenção das altas coberturas vacinais e a ampliação da vigilância e das recomendações de investigação e tratamento da inf ecção latente pelo Mycobacterium tuberculosis (BRASIL, 2020). O imunobiológico utilizado para prevenção das formas graves da TB é a vacina Bacilo de CalmetteGuérin (BCG) e seu esquema de vacinação corresponde à dose única administrada o mais precocemente possível (BRASIL, 2014).

A vacina BCG foi desenvolvida entre 1906 e 1919 porCamille Calmette e Albert Guerin no Instituto Pasteur em Paris, França. Ela constitui uma importante forma de proteção contra formas graves de tuberculose (meníngea e miliar) em menores de cinco anos, causadas pelo Mycobacterium tuberculosis em países onde ainda ocorre. A aplicação também é recomendada a contatos domiciliares de portadores hanseníase, como forma de proteção contra Mycobacterium leprae (BRASIL, 2019a; BRASIL, 2020) e oferece um bom nível de proteção, quando disponibilizada nos primeiros dias de vida, sendo perceptível o valor de sua eficácia na diminuição do desenvolvimento da doença, quando em comparação àqueles que não receberam a vacina (REIS AB, et al., 20109).

Sua constituição contém bacilos vivos, a partir de cepas do Mycobacterium bovis e a sua via de administração é intradérmica. Ela induz o tipo retardado de reação de hipersensibilidade e imunidade mediada por células no hospedeiro no período de 4 a 8 semanas após a imunização. Alterações são observadas no local da vacinação, na forma de pápula, pústula, úlcera e cicatriz, possuindo uma eficácia que varia de 0 a 80\% (BRASIL, 2014; SAROHA M, et al., 2015).

A vacinação conta com contraindicações, sendo elas: indivíduos a partir de cinco anos, portadores de imunodeficiência congênita ou adquirida, mesmo que assintomáticos e sem sinais de imunodeficiência, pacientes com neoplasias malignas, pacientes em uso de doses elevadas de corticosteróides por mais de duas semanas e outras drogas imunodepressoras (BRASIL, 2014).

Cabe destacar que desde 2019, a partir do posicionamento da Organização Mundial da Saúde (OMS), o PNI passou a não indicar a revacinação de crianças que não desenvolveram cicatriz vacinal após a administração da BCG, diante da inexistência de evidências científicas que justificassem esse procedimento (BRASIL, 2019b). 
No início da pandemia do novo coronavírus, observou-se que nos países em que a vacina fazia parte do calendário vacinal, a incidência e a mortalidade eram menores do que em países que não tinham esse imunobiológico disponível em seus programas de vacinação. As análises confirmaram essa relação positiva, mas atribuída a outros fatores, como a genética na população, o tipo de cepa da vacina BCG, o nível de saúde e riqueza, a política de migração, comorbidades e ações de distanciamento social. Vários estudos estão sendo realizados para comprovar esse benefício e, no entanto, a Organização Mundial da Saúde (OMS) ainda não recomenda a vacinação com BCG para a prevenção do COVID-19 (REKA G, et al., 2020).

Assim, considerando o aumento dos casos da doença no município de Porto Alegre e o baixo índice de cura, no ano de 2019 foi definida pelo Plano Municipal de Enfrentamento da Tuberculose, a meta de implantação da administração da vacina BCG nas maternidades do município. Essa estratégia buscou facilitar o acesso ao imunobiológico, aumentar a Cobertura Vacinal (CV) e, com isso, diminuir os casos associados às formas mais graves (PREFEITURA MUNICIPAL DE PORTO ALEGRE, 2019).

O sucesso desse processo de mudança é considerado fundamental, visto que a cidade apresenta o quarto maior índice de incidência da doença entre as capitais, alta taxa de abandono de tratamento (24\%) e uma taxa de cura de 56\% (PREFEITURA MUNICIPAL DE PORTO ALEGRE, 2019).

Diversas pesquisas foram realizadas para analisar a proteção após a administração da vacina BCG, encontrando em média, um ef eito entre $16 \%$ e $73 \%$. Já, quando consideramos as formas de tuberculose, para TB pulmonar, o nível foi de 10 a $66 \%$ e para as formas graves (meníngeas e militares) os índices superaram os 50\% (BARRETO M, et al., 2006).

Assim, esse estudo teve como objetivo escrever o processo de implementação da administração da vacina BCG nas maternidades no município de Porto Alegre, Rio Grande do Sul e os ef eitos dessa estratégia. Vale destacar que não foram encontradas pesquisas semelhantes, demonstrando a relevância da discussão dessa temática e o ineditismo do registro dos dados investigados.

\section{MÉTODOS}

A pesquisa quantitativa é realizada através da coleta e análise de dados quantitativos sobre variáveis. Este tipo de estudo é capaz de identificar as realidades e suas dinâmicas. Para muitos é necessário utilizar as ferramentas da pesquisa quantitativa para a avaliação da qualidade do cuidado e dos programas de saúde. Por isso, a relevância do conhecimento desse tipo de pesquisa (ESPERÓN JMT, 2017).

Esse trabalho apresenta uma descrição das ações realizadas pelo Núcleo de Imunizações e de uma avaliação dos impactos gerados pela implantação da administração da vacina BCG nas maternidades do município de Porto Alegre.

Para operacionalizar esse projeto foram organizadas ao longo do ano diversas capacitações teóricas e práticas com os profissionais dos serviços de saúde materno-infantil, de hospitais privados e públicos. A observação sistemática do novo fluxo fez surgir o questionamento que originou o problema desta pesquisa: quais foram os impactos gerados pela implantação da administração da vacina BCG nas maternidades?

Para a análise foram empregados os dados extraídos do Sistema de Informação do Programa Nacional de Imunizações (SI-PNI) e do Sistema de Insumos Estratégicos em Saúde (SIES), sendo organizados e tabulados em uma planilha, utilizando-se o software Microsoft Exce ${ }^{\circledR} 2010$.

O SI-PNI é um sistema de informação instituído pelo Ministério da Saúde. Ele possui o diferencial de ser o primeiro sistema nominal, que gera um prontuário para cada indivíduo vacinado e que pode ser acessado por todas as salas de vacinas pertencentes ao território nacional. Ainda, constitui-se um notável recurso de gestão, gerando informações que servem para o planejamento, organização e tomada de decisão das instâncias federais, estaduais e municipais (SILVA BS, et al., 2018).

Esse sistema está em uso desde o ano de 2017 no município de Porto Alegre, capital do estado do Rio Grande do Sul (RS). A implementação se deu inicialmente pelas salas de vacinas com maior demanda, sendo 
ampliada posteriormente às demais. Atualmente, é alimentado através das digitações dos serviços públicos (unidades de saúde, Centro de Referência de Imunobiológicos Especiais e hospitais) e privados (hospitais, clínicas de vacinação e farmácias). Através dele podem ser emitidos diversos relatórios como cobertura vacinal, doses aplicadas, data da administração, boletim de comparecimento, campanhas de vacinação e monitoramento dos imunobiológicos.

O SIES é um sistema que administra o recebimento e a distribuição das vacinas, sendo utilizado para acompanhar os quantitativos recebidos e os locais para onde foram entregues, bem como monitoramento de estoque. Os dados referentes à cobertura vacinal e a faixa etária na data da administração foram extraídos do SI-PNI. Os dados sobre a idade do vacinado foram retirados do relatório de vacinados por vacina, e tabulados posteriormente em uma planilha. O SIES forneceu os quantitativos de imunobiológico distribuídos nos anos de 2019 e 2020, assim como os locais para onde foram disponibilizados.

Para a construção do estudo foram definidas as seguintes etapas: elaboração da pergunta norteadora, busca na literatura para referencial teórico, coleta de dados, análise crítica e discussão dos resultados, delineamento e redação da revisão.

A pesquisa foi realizada com os dados do município de Porto Alegre, capital do estado do Rio Grande do Sul, população estimada de 1.413.094 habitantes, Índice de Desenvolvimento (IDH) elevado de 0,865 e um Produto Interno Bruto (PIB) per capita de R\$ 25.712,62 mil (PREFEITURA DE PORTO ALEGRE, 2021a).

A cidade disponibiliza um total de 143 serviços de saúde que compõem a atenção primária, sen do divididos entre Unidades Básicas de Saúde (UBS), 55 e Unidades de Saúde da Família (USF) 88 (PREFEITURA DE PORTO ALEGRE, 2021b). O estudo utilizou somente dados secundários, não sendo necessária a avaliação do Comitê de Ética e Pesquisa.

\section{RESULTADOS E DISCUSSÃO}

A cobertura vacinal é um importante indicador que auxilia no direcionamento das intervenções em diferentes instâncias, sendo elas federais, estaduais ou municipais. Quando adequadas, tem-se o controle ou a probabilidade de eliminação ou erradicação de doenças imunopreveníveis sob vigilância. Uma cobertura vacinal adequada protege também aqueles que por alguma razão, não podem se vacinar (BRASIL, 2015; FUNDAÇÃO OSWALDO CRUZ, 2020; SOCIEDADE BRASILEIRA DE IMUNIZAÇÕES, 2021).

Na Tabela 1, está descrita a série histórica da vacina BCG no município de Porto Alegre, segundo o Sistema de Informação do Programa Nacional de Imunizações (SI-PNI) entre os anos de 2015 e 2020, considerando a população de menores de um ano de idade.

Tabela 1 - Série histórica da cobertura vacinal da BCG, no município de Porto Alegre - RS, em menores de um ano, período $2015-2020$.

\begin{tabular}{cccc}
\hline Ano & População & Doses Aplicadas & Cobertura Vacinal (\%) \\
\hline 2015 & 19.739 & 17.671 & 89,5 \\
2016 & 19.188 & 17.757 & 92,5 \\
2017 & 19.188 & 18.427 & 96,0 \\
2018 & 18.635 & 16.446 & 88,3 \\
2019 & 18.490 & 15.724 & 85,0 \\
2020 & 18.490 & 18.501 & 100,1 \\
\hline
\end{tabular}

Fonte: Capponi RL, et al, 2021; dados extraídos do Ministério da Saúde/Secretaria de Vigilância em Saúde/Coordenadoria Geral do Programa Nacional de Imunizações/Sistema de Informações do Programa Nacional de Imunizações, 2021.

Pode-se observar que a cobertura vacinal, após uma elevação em 2017, se encontrava em um movimento de queda, estando abaixo dos índices encontrados nos anos anteriores e também da cobertura recomendada pelo Ministério da Saúde, de 90\% (BRASIL, 2015). A queda no número da população alvo, que é visualizada 
através uma diminuição constante no número de nascimentos, é semelhante à tendência encontrada em quase todo território nacional (PREFEITURA DE PORTO ALEGRE, 2021c).

No ano de 2020, após as modificações nos serviços de vacinação, os valores demonstram um aumento significativo de recém-nascidos vacinados em comparação ao ano de 2019, estando acima do quantitativo preconizado ao atingir o valor de $100 \%$ de cobertura vacinal $(p<0,0001)$.

O alcance de uma cobertura vacinal de 100\% demonstra que o município está aplicando um quantitativo de vacinas maior do que a população alvo estipulada. Isso acontece devido ao fato de Porto Alegre ser referência estadual para o atendimento ao pré-natal de alto risco e de um histórico de assistência às urgências e emergências neonatais e pediátricas, compreendendo uma área da atenção terciária e englobando a realização de procedimentos com diferentes níveis de complexidade.

Sendo a vacinação um procedimento que não pode ser restringido de acordo com a residência do usuário, as maternidades são orientadas a imunizar todos os recém-nascidos atendidos, desde que apresentem as condições recomendadas para a aplicação. Essas doses são digitadas no Sistema de Informação do PNI e até o momento englobam a CV do município que realizou a aplicação.

Por isso, seria essencial que o Ministério da Saúde atualizasse o Sistema de Informação para que somente as crianças vacinadas residentes fossem consideradas para análise da cobertura vacinal, fazendo com que a avaliação desse dado seja mais precisa e correta para realização da busca ativa dos faltosos. Mesmo assim, pode-se perceber que a mudança no fluxo e no processo de trabalho feita nos serviços vacinadores foi exitosa e mantém a CV contra tuberculose elevada e acima da meta estipulada pelo PNI.

Vale ressaltar que são mantidas algumas unidades de saúde de referência para administração da vacina BCG, distribuídas entre os distritos de saúde, considerando que algumas crianças podem possuir contraindicações no momento da internação hospitalar, sendo indicada a aplicação mais tardiamente ou após a resolução do caso. Essas unidades, também funcionam como referência para os pacientes que realizam tratamento contra a hanseníase e possuem indicação da imunização.

Outro aspecto a ser considerado é o período entre o nascimento da criança e a administração do imunobiológico. O desabastecimento da vacina em 2018 fez com que o município elencasse unidades de referência para a aplicação. Isso acarretou a dificuldade de acesso ao imunobiológico, tendo em vista que o cidadão tinha que se deslocar até outra unidade de saúde em dias e horários pré-definidos. Essa dificuldade era influenciada pelas condições socioeconômicas da família, que muitas vezes acabava adiando a imunização por não conseguir realizar o deslocamento necessário.

Observando a idade do recebimento da vacina BCG entre os anos de 2019 e 2020, percebe-se que em 2019, quando ainda não havia o fluxo nas maternidades, grande parte das aplicações acontecia entre o $3^{\circ} \mathrm{e}$ o $30^{\circ}$ dia de vida $(61,9 \%)$. Já no ano de 2020 , os recém-nascidos em sua maioria foram imunizados nos primeiros dias de vida, atingindo um percentual de $85,9 \%$ vacinados até o $2^{\circ}$ dia após o nascimento $(p<0,0001)$. Deve-se observar, também, a diminuição expressiva do quantitativo de doses realizadas entre 0 $31^{\circ}$ dia e até o primeiro ano de idade, apresentando uma queda de $8 \%$ nas aplicações tardias, como mostra a Tabela 2.

Tabela 2 - Frequência da administração da vacina BCG segundo faixa etária (até um ano de vida, entre o 2ำ e o 30ㅇ dia de vida e abaixo de um ano de idade), Porto Alegre - RS, anos 2019 e 2020.

\begin{tabular}{ccc}
\hline Faixa Etária & $\mathbf{2 0 1 9}$ & $\mathbf{2 0 2 0}$ \\
\hline Administrações até o $2^{\circ}$ dia de vida & $4.182(26,6 \%)$ & $15.897(85,9 \%)$ \\
Administrações entre o $2^{\circ}$ e o $30^{\circ}$ dia & $9.737(61,9 \%)$ & $2.102(11,4 \%)$ \\
Administração até um ano de vida & $1.805(11,5 \%)$ & $502(2,7 \%)$ \\
\hline Total de administrações & $\mathbf{1 5 . 7 2 4}(\mathbf{1 0 0 , 0 \% )}$ & $\mathbf{1 8 . 5 0 1 ( 1 0 0 , 0 \% )}$ \\
\hline
\end{tabular}

Fonte: Capponi RL, et al, 2021; dados extraídos do Ministério da Saúde/Secretaria de Vigilância em Saúde/Coordenadoria Geral do Programa Nacional de Imunizações/Sistema de Informações do Programa Nacional de Imunizações, 2021. 
Assim, se confirma que a centralização da vacinação contra tuberculose nas maternidades, garantiu fácil acesso dos recém-nascidos ao imunobiológico e a imunização nas primeiras horas de vida, conforme o preconizado pelo Ministério da Saúde (BRASIL, 2014).

Na Tabela 3 estão os dados sobre o perfil do serviço vacinador, em que se observa uma modificação nos locais responsáveis pela aplicação da vacina BCG. A administração que era realizada nas unidades de saúde de referência em $2019(68,5 \%)$ passou a ser centralizada nas maternidades, atingindo um percentual de $78,4 \%$ nos hospitais públicos e de $14,8 \%$ nos hospitais privados $(p<0,0001)$.

Sobre as maternidades dos setores privados é essencial destacar que rotineiramente a oferta desse serviço é feita com os imunobiológicos adquiridos diretamente dos laboratórios produtores autorizados pelo Ministério da Saúde, sendo o custo da vacina e da aplicação repassado ao usuário. Caso o mesmo opte por não realizar a imunização no hospital, a orientação é procurar as unidades de saúde de referência do município. Alguns dos hospitais privados já disponibilizavam a BCG ao seu público antes das alterações.

No ano de 2020 ocorreu um desabastecimento da vacina BCG nos hospitais e clínicas de vacinação privados, com queda do número de aplicações de 2019 , passando de $28,7 \%$ e $2,8 \%$ para $14,8 \%$ e $1,4 \%$, respectivamente. Quando essa situação acontece, o Núcleo de Imunizações proporciona a possibilidade de a maternidade receber as vacinas e os insumos oriundos do Programa Nacional de Imunizações, mantendo assim, a imunização o mais precoce possível. Nesses casos, a maternidade se compromete a não realizar a cobrança desse serviço, mantendo o fluxo estipulado.

É importante salientar que nos períodos em que a maternidade privada utiliza os imunobiológicos do PNI as informações digitadas passam a ser incluídas como serviços públicos no Sistema de Informação.

Tabela 3 - Distribuição das doses administradas da vacina BCG, por tipo de serviço vacinador, Porto Alegre - RS, anos 2019 e 2020.

\begin{tabular}{ccc}
\hline Tipo de Serviço Vacinador & $\mathbf{2 0 1 9}$ & $\mathbf{2 0 2 0}$ \\
\hline Unidades de Saúde & $10.777(68,5 \%)$ & $989(5,4 \%)$ \\
Hospitais Privados & $4.510(28,7 \%)$ & $2.742(14,8 \%)$ \\
Hospitais Públicos & $0(0,0 \%)$ & $14.510(78,4 \%)$ \\
Clínicas de Vacinação & $437(2,8 \%)$ & $260(1,4 \%)$ \\
\hline Total de administrações & $\mathbf{1 5 . 7 2 4 ( 1 0 0 , 0 \% )}$ & $\mathbf{1 8 . 5 0 1 ( 1 0 0 , 0 \% )}$ \\
\hline
\end{tabular}

Fonte: Capponi RL, et al, 2021; dados extraídos do Ministério da Saúde/Secretaria de Vigilância em Saúde/Coordenadoria Geral do Programa Nacional de Imunizações/Sistema de Informações do Programa Nacional de Imunizações, 2021.

Desde 2018, o Ministério da Saúde tem distribuído vacina BCG de dois laboratórios. O laboratório brasileiro Fundação Ataulpho de Paiva (FAP), que possui como dosagem $0,1 \mathrm{~mL}$ independentemente da idade e um frasco com apresentação de 10 doses e o do laboratório Serum Institute of India Ltd - dose de 0,05mL em menores de um ano e de $0,1 \mathrm{~mL}$ em maiores de um ano e apresentação de 20 doses por frasco (BRASIL, 2014).

Até meados do ano de 2019 se recebia rotineiramente o imunobiológico do laboratório FAP. Em 2020 foi introduzida a vacina do laboratório Serum. Na Tabela 4 está o quantitativo de doses distribuídas no município pelo Núcleo de Imunizações entre os anos de 2019 e 2020 de acordo com a apresentação do frasco. Percebese que o número de doses distribuídas em 2020 dobrou em comparação ao ano de 2019. Esse fato se deve a algumas variáveis que devem ser consideradas para a análise da eficiência da modificação do fluxo nas unidades vacinadoras.

Após a capacitação, algumas maternidades optaram por realizar a administração da vacina junto com o imunobiológico da Hepatite B no Centro Obstétrico (CO). Contudo, foi evidenciado aos poucos que a melhor estratégia era a aplicação no Alojamento Conjunto (AC) após a alta do recém-nascido. Quando a BCG é realizada no AC, todas as crianças que são liberadas naquele dia são agendadas para receberem a aplicação, 
otimizando assim, o uso do imunobiológico que possui duração de 6 horas após o frasco ser aberto. Já no $\mathrm{CO}$, o número de aplicações dependia da quantidade de nascidos vivos naquele momento.

A vacina BCG é administrada pela via intradérmica, pouco usual na rotina. Assim, os profissionais necessitam de capacitações periódicas e de tempo para se adequarem à introdução da nova técnica. A falta de experiência com a via de administração pode estar relacionada com o desperdício do imunobiológico.

O relato dos profissionais que atuam nas salas de vacinas, de que o frasco da vacina BCG não disponibiliza o quantitativo correto de doses que deveria após a diluição já acontecia quando o laboratório utilizado era a FAP. Porém, a perda referida era pequena (uma média de 2-3 doses), considerando que o frasco deveria render 10 doses. Com a introdução do laboratório Serum, ocorreu um aumento considerável, pois segundo alguns profissionais a média de doses disponíveis em um frasco variava entre 11 a 17 doses de um frasco que deveria possuir 20 doses. Obteve-se tal informação através de relatos de vacinadores e teste realizado na sede do Núcleo de Imunizações de Porto Alegre, onde após a diluição correta do frasco, e aspiração de doses, o mesmo rendeu somente 11 doses.

Tabela 4 - Número de doses da vacina BCG distribuídas entre 2019 e 2020 no município de Porto Alegre RS, anos 2019 e 2020.

\begin{tabular}{ccc}
\hline Tipo de frasco & Número de doses distribuídas por ano \\
\hline Ano & $\mathbf{2 0 1 9}$ & $\mathbf{2 0 2 0}$ \\
\hline Frasco de 10 doses & 18.940 & 0 \\
Frasco de 20 doses & 16.140 & 66.340 \\
\hline Total de doses & $\mathbf{3 5 . 0 8 0}$ & $\mathbf{6 6 . 3 4 0}$ \\
\hline
\end{tabular}

Fonte: Capponi RL, et al, 2021; dados extraídos de Sistema de Insumos Estratégicos de Saúde, 2021.

\section{CONCLUSÃO}

Os dados encontrados demonstraram que a mudança no fluxo dos serviços vacinadores oportunizou aumento na cobertura vacinal do município que apresentava queda no biênio 2018-2019, atingindo uma porcentagem de $100 \%$ após a alteração. Com o novo fluxo, a maioria dos recém-nascidos encontra-se imunizada até $02^{\circ}$ dia de vida, período mais próximo da meta preconizada pelo Ministério da Saúde, diferentemente do que estava acontecendo com a centralização dos serviços de administração nas unidades de saúde de referência. Assim, as mudanças realizadas trouxeram um maior acesso ao imunobiológico da BCG. Os quantitativos de doses distribuílas quase dobraram em relação ao que foi entregue em 2019. Contudo, faz necessário destacar a modificação do laboratório produtor e o relato dos profissionais sobre as perdas técnicas encontradas no frasco de 20 doses. Por isso e por se tratar de uma investigação inédita e relevante para a manutenção das coberturas vacinais, como sugestão, acredita-se ser interessante a continuidade da avaliação das informações verificadas nos próximos anos, objetivando avaliar possíveis melhorias para qualificação do processo de trabalho e afirmar a importância da imunização como prática de prevenção em Saúde Pública.

\section{REFERÊNCIAS}

1. BARRETO ML, et al. Vacina BCG: eficácia e indicações da vacinação e da revacinação. Jornal de Pediatria, 2006; 82: 445-54.

2. BRASIL. Boletim Epidemiológico da Tuberculose. 2020. Disponível em: https://www.gov.br/saude/pt-br/centrais-deconteudo/boletim-tuberculose-2020-marcas-1-pdf. Acessado em:22 de nov. de 2021.

3. BRASIL. Coberturas Vacinais no Brasil: período 2010-2014. 2015. Disponível em: https://siteal.iiep.unesco.org/sites/default/files/sit_accion_files/br_5113.pdf. Acessado em:25 de out. de 2021.

4. BRASIL. Dados do Instituto Brasileiro de Geografia e Estatística (IBGE) - Porto Alegre. Disponível em: https://cidades.ibge.gov.br/brasil/rs/porto-alegre/panorama. Acessado em: 12 de set. de 2021.

5. BRASIL. Guia de Vigilância em Saúde. 2019a. Disponível em: https://bvsms.saude.gov.br/bvs/publicacoes/guia_vigilancia_saude_3ed.pdf. Acessado em:15 de out. de 2021.

6. BRASIL. Manual de Normas e Procedimentos para Vacinação. 2014. Disponível em: https://bvsms.saude.gov.br/bvs/publicacoes/manual_procedimentos_vacinacao.pdf. Acessado em: $7 \mathrm{de}$ dez. de 2021. 
7. BRASIL. Manual de Vigilância Epidemiológica de Eventos Adversos Pós-vacinação. 2020. Disponível em: https://bvsms.saude.gov.br/bvs/publicacoes/manual_vigilancia_epidemiologica_eventos_vacinacao_4ed.pdf. Acessado em: 15 de out. de 2021.

8. BRASIL. Manual técnico para o controle da tuberculose: cadernos de atenção básica. 2002. Disponível em: https://bvsms.saude.gov.br/bvs/publicacoes/manual_tecnico_controle_tuberculose_cab6.pdf. Acessado em: $18 \mathrm{de}$ out. de 2021.

9. BRASIL. Nota Informativa n $^{\circ}$ 10/2019. 2019b. Disponível em: https://sbim.org.br/images/files/notas-tecnicas/notainformativa-10-2019-cgpni.pdf. Acessado em: 19 de nov. de 2021.

10. DOMINGUES CMAS, et al. 46 anos do Programa Nacional de Imunizações: uma história repleta de conquistas e desafios a serem superados. Cadernos de Saúde Pública, 2020;36:1-17.

11. ESPERÓN JMT. Pesquisa quantitativa na ciência da enfermagem. Escola Anna Nery, 2017;21:1-2.

12. FUNDAÇÃO OSWALDO CRUZ. Vacinas e vacinação no Brasil: horizontes para os próximos 20 anos. $1^{\underline{a}}$ ed. Rio de Janeiro:Edições Livres, 2020;250p.

13. PREFEITURA MUNICIPAL DE PORTO ALEGRE. Nascidos Vivos em Porto Alegre. 2021c. Disponível em: https://pentahopmpa.procempa.com.br/pentaho/api/repos/\%3Apublic\%3ASMS\%3Alndicadores\%3AEventos\%20Vitais\%3Anascido s\%20vivos.wcdf/generatedContent?ts=1583865946734. Acesso em: 10 de out. de 2021 .

14. PREFEITURA MUNICIPAL DE PORTO ALEGRE. Plano Municipal de Enfrentamento da Tuberculose. 2019. Disponível em: http://proweb.procempa.com.br/pmpa/prefpoa/cms/usu_doc/plano_tuberculose.pdf. Acesso em: 25 de set. de 2021.

15. PREFEITURA MUNICIPAL DE PORTO ALEGRE. Serviços da Secretaria de Saúde. 2021b. Disponível em: https://prefeitura.poa.br/carta-de-servicos. Acesso em:9 de out. de 2021.

16. REIS AB, et al. A eficácia da vacina BCG no controle da tuberculose em recém nascidos. Ciências Biológicas e de Saúde Unit, 2019;5(3): 95-104.

17. REKA G, et al. The influence of vaccination against tuberculosis with the Bacillus-Calmette-Guérin (BCG) vaccine on COVID-19 incidence and mortality - review of the literature. Przegląd Epidemiologiczny, 2020; 74(2): 290-302.

18. SAROHA M, et al. Immunogenicity and safety of early vs delayed BCG vaccination in moderately preterm (31-33 weeks) infants. Human Vaccines \& Immunotherapeutics, 2015;11 (12):2864-2871.

19. SECRETARIA DE VIGILÂNCIA EM SAÚDE (SVS). In: Datasus. Sistema de Insumos Estratégicos em Saúde (SIES). Disponível em: http://sies.saude.gov.br/. Acessado em:25 de nov. de 2021.

20. SILVA BS, et al. Estudo de avaliabilidade do Sistema de Informação do Programa Nacional de Imunização. Revista Brasileira de Enfermagem, 2018;71(Supl.1):615-624.

21. SOCIEDADE BRASILEIRA DE IMUNIZAÇÕES. 2021. In: Vacinas. Conceitos Importantes. Disponível em: https://familia.sbim.org.br/vacinas/conceitos-importantes. Acesso em: 11 de abr. de 2021. 